allocated to either a 'no-feedback' or 'feedback' group, and performed 60 seconds of two-thumb (TT) and two-finger (TF) chest compressions on a "physiological" CPR manikin instrumented to measure chest deflections. Baseline data were recorded for both groups without feedback, before chest compressions were repeated in the experimental phase with the 'feedback' group receiving real-time performance feedback. Chest compression depths, chest release forces, chest compression rates and compression duty cycles were recorded for all participants. Quality indices were calculated to report the proportion of chest compressions that achieved internationally recommended quality targets for each measure, with an overall quality index calculated to report the proportion of chest compressions that simultaneously achieved all four quality targets. Results were compared between the 'no-feedback' and 'feedback' groups.

Results Baseline data were consistent with other studies, with $<\%$ of chest compressions simultaneously achieving all four internationally recommended quality targets. During the experimental stage (Table 1), the provision of real-time performance feedback improved the quality of the chest compression depths, chest compression rates and compression duty cycles provided by both techniques (all measures: $p<0.001$ ). This enabled the 'feedback' group to simultaneously achieve all four quality targets in $75 \%$ of TF and $80 \%$ of TT technique chest compressions, whilst $<1 \%$ of chest compressions achieved this for the 'no-feedback' group.

Conclusions Real-time performance feedback considerably increased the quality of chest compressions provided during simulated infant CPR. If these results transfer into clinical practise this technology could, for the first time, support resuscitators in performing high quality chest compressions during infant CPR and thus potentially improve future outcomes.

\section{P12 CHILD DEATHS DUE TO INJURY IN FOUR UK COUNTRIES: 1980 TO 2010}

doi:10.1136/archdischild-2013-304107.012

P Hardelid, J Davey, N Dattani, R Gilbert. MRC Centre of Epidemiology for Child Health, UCL Institute of Child Health, London, UK

Aims To examine trends in child deaths due to injury England, Scotland, Wales and Northern Ireland between 1980 and 2010.

Methods Data from death certificates from children who died aged 28 days to 18 years between 1980 and 2010 were obtained from the national statistics agencies in the four countries. Injury deaths, including poisoning, was defined by an external cause code (from the International Classification of Diseases) recorded as the underlying cause of death. We estimated rates of injury deaths per 100,000 resident children by sex, age group (28 days to nine years, and 10 to 18 years), time period, country of residence and type of injury (accidental or non-accidental). Mortality rates were adjusted for reporting delay.

Results Child mortality due to injury has declined in all four countries of the UK. England consistently experienced the lowest mortality rate throughout the study period. For children aged 28 days to nine years, differences in mortality rates between England and the other three countries declined over the study period, whereas for children aged 10 to 18 years, differences in mortality rates increased. Inter-country differences were largest for boys aged 10 to 18 years with mortality rate ratios of 1.34 (95\% confidence interval 1.13 , 1.60) for Wales, $1.66(1.46,1.89)$ for Scotland and 1.83 (1.52, 2.21) for Northern Ireland compared to England (the baseline). The decline in mortality due to injury was accounted for by a decline in accidental deaths; no declines were observed for any age groups for non-accidental deaths, that is deaths caused by self-harm, assault or from undetermined intent.

Conclusions Whilst child deaths from injury have declined in all four UK countries, substantial differences in mortality rates remain between countries, particularly for older boys. This group stands to gain most from policy interventions to reduce deaths from injury and poisoning in children.

\section{P13 'EFFORT OF BREATHING' IS NOT AN IMPORTANT PARAMETER IN A PAEDIATRIC EARLY WARNING SCORING SYSTEM}

doi:10.1136/archdischild-2013-304107.013

LL Sinitsky, A Reece. Department of Paediatrics, Watford General Hospital, Watford, UK

Aims Across the UK there is diverse practise in the use of Paediatric Early Warning Scores (PEWS). Many scoring systems are in use and include different physiological parameters to identify children at risk of life-threatening deterioration. Unlike adult practise, PEWS often comprise of both objective and subjective criteria. 'Effort of breathing' is a subjective parameter commonly included in paediatric scoring systems. Determining a child's effort of breathing is influenced by factors including appropriate exposure of the patient as well as clinical skill, experience and acumen of the scorer.

As part of a study assessing the validity of PEWS charts, a large data set was collected. Analysis of the NHS Institute PEWS chart is made here.

Method Physiological parameters were collected retrospectively from a cohort of 1537 children aged 0-16 years attending a district general hospital's Children's Emergency Department over a 5 week period. Admission to Paediatric High Dependency or Intensive Care were used as proxy outcome measures for serious and life-threatening deterioration.

Results Data was complete for 967 records. At a best cut-off score of 3 , NHS Institute PEWS had a sensitivity of $64.3 \%$ (95\% CI 35.6 86.0 ), specificity of $96.5 \%$ (95\% CI 95.1-97.6), positive predictive value of $21.4 \%$ (95\% CI 10.8-37.2) and negative predictive value of 99.5\% (95\% CI 98.7-99.8). The area under the Receiver Operating Characteristic curve (AUC) (figure 1) was 0.86 (95\% CI 0.74-0.98, $\mathrm{p}<0.01$ ). If 'effort of breathing' was excluded from NHS Institute PEWS the AUC was 0.85 (95\% CI 0.74-0.97, p < 0.00).

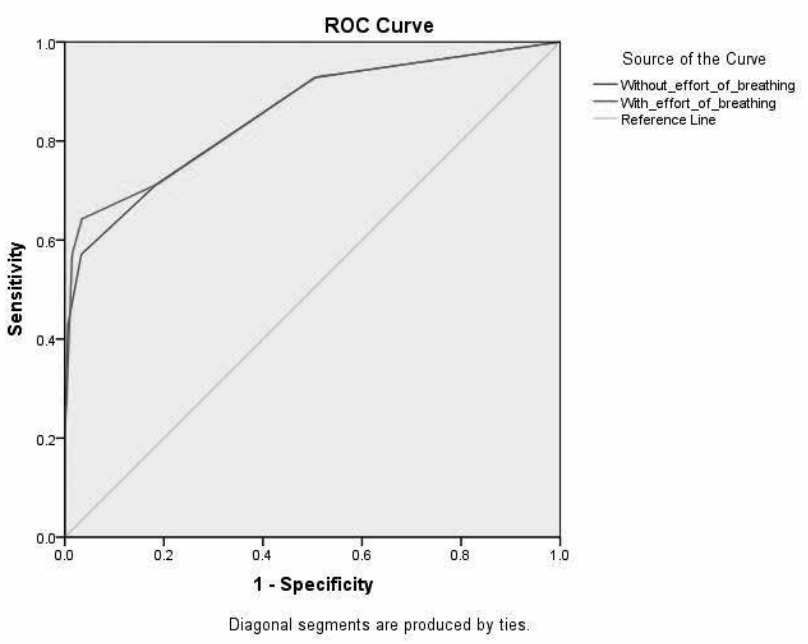

Abstract P13 Figure 1 Receiver operating characteristic curve for NHS Institute PEWS with and without effort of breathing.

Conclusion The NHS Institute PEWS is a valid tool with good diagnostic accuracy in recognising children at risk of serious and life-threatening deterioration at triage in the Emergency Department. The predictive power did not change when 'effort of breathing' was excluded. It is reassuring that such a subjective parameter does not undermine the value of the scoring system. However, further work is needed to determine whether other subjective measures have any value in paediatric early warning tools. 